\title{
A proof of a transformation formula in the theory of partitions.
}

\author{
By Kanesiroo IsEKI
}

(Received June 8, 1951)

Let us denote by $p(n)$ the number of unrestricted partitions of a positive integer $n$. Then we have

$$
f(x)=1+\sum_{n=1}^{\infty} p(n) x^{n}=\prod_{n=1}^{\infty} \frac{1}{1-x^{n}},
$$

where $f(x)$ is defined and regular for $|x|<1$. Now this function $f(x)$ is known to satisfy a remarkable transformation formula (see Rademacher [3] ) :

$$
f\left(e^{\frac{2 \pi i h}{k}-\frac{2 \pi z}{k}}\right)=\omega_{h, k} \sqrt{z} e^{\frac{\pi}{12 k z}-\frac{\pi z}{12 k}} f\left(e^{\frac{2 \pi i H}{k}-\frac{2 \pi}{k z}}\right),
$$

where $h, k$ and $H$ are positive integers such that

$$
(h, k)=1, \quad h H \equiv-1(\bmod k),
$$

and

$$
\omega_{h, k}=\exp \left(\pi i \sum_{m=1}^{k-1} \frac{m}{k}\left(\frac{h m}{k}-\left[\frac{h m}{k}\right]-\frac{1}{2}\right)\right),
$$

an empty sum meaning zero; further, $z$ is a complex variable with positive real part and we take the principal branch as the determination of $\sqrt{z}$.

The formula (1) was used by Hardy-Ramanujan, and also by Rademacher subsequently, in their famous researches [2] and [4] on the function $p(n)$.

It is the main object of this paper to give a proof of (1) which is directly based on the following well known transformation formula in the theory of elliptic theta-functions:

$$
\sum_{n=-\infty}^{\infty} e^{-(n+\alpha)^{2} \pi t}=\frac{1}{\sqrt{ } t} \sum_{n=-\infty}^{\infty} e^{-\frac{n^{2} \pi}{t}} \cos (2 \pi n \alpha),
$$


where $\alpha$ is real and $t>0$.

Let us begin by defining a branch of the logarithm of $f(x)$ for $|x|<1$ by

$$
F(x)=\log f(x)=\sum_{n=1}^{\infty} \log \frac{1}{1-x^{n}}=\sum_{m=1}^{\infty} \sum_{n=1}^{\infty} \frac{x^{m n}}{m}
$$

where we take

$$
\log \frac{1}{1-x^{n}}=\sum_{m=1}^{\infty} \frac{x^{m n}}{m}
$$

We put

$$
x=e^{\frac{2 \pi i h}{k}-\frac{2 \pi z}{k}}, \quad \tilde{x}=e^{\frac{2 \pi i H}{k}-\frac{2 \pi}{k z}} .
$$

Then, to prove (1), it suffices to show that

$$
\begin{gathered}
F(x)=\Omega i+\frac{1}{2} \log z+\frac{\pi}{12 k z}-\frac{\pi z}{12 k}+F(\tilde{x}), \\
\Omega=\Omega_{h, k}=\pi \sum_{m=1}^{k-1} \frac{m}{k}\left(\frac{h m}{k}-\left[\frac{h m}{k}\right]-\frac{1}{2}\right),
\end{gathered}
$$

where in (3) we take the principal branch as the determination of $\log z$.

We may assume $z>0$, for the general case of $z$ with positive real part is reduced to this case by the theory of analytic continuation. We shall prove (3) by decomposing it into its real and imaginary parts :

$$
\begin{aligned}
\Re F(x)= & \frac{1}{2} \log z+\frac{\pi}{12 k z}-\frac{\pi z}{12 k}+\Re F(\tilde{x}), \\
& \Im F(x)=\Omega+\Im F(\tilde{x}) .
\end{aligned}
$$

To establish (5), we start from the integral

$$
\int_{0}^{\infty} e^{-t^{2}-\frac{a^{2}}{t^{2}}} d t=\frac{\sqrt{\pi}}{2} e^{-2 a} \quad(a>0),
$$

which is an easy consequence of

$$
\int_{0}^{\infty} e^{-t^{2}} d t=\frac{\sqrt{\pi}}{2}
$$

We transform the above integral into the following form: 


$$
\int_{0}^{\infty} e^{-a^{2} t^{2}-\frac{b^{2}}{t^{2}}} d t=\frac{\sqrt{\pi}}{2 a} e^{-2 a b} \quad(a>0, b>0)
$$

Putting $a=\sqrt{\pi} m z / k$ and $b=\sqrt{\pi} n$, where $m$ and $n$ are positive integers, we find

$$
\frac{1}{m} e^{-\frac{2 \pi m n z}{k}}=\frac{2 z}{k} \int_{0}^{\infty} e^{-\frac{\pi m^{2} z^{2} t^{2}}{k^{2}}-\frac{\pi n^{2}}{t^{2}}} d t
$$

Hence, by absolute convergence,

$$
F(x)=\sum_{m=1}^{\infty} \sum_{n=1}^{\infty} \frac{1}{m} e^{m n\left(\frac{2 \pi i h}{k}-\frac{2 \pi z}{k}\right)}=\frac{2 z}{k} \int_{0}^{\infty} \sum_{m=1}^{\infty} \sum_{n=1}^{\infty} e^{\frac{2 \pi i h m n}{k}-\frac{\pi m^{2} z^{2} t^{2}}{k^{2}}-\frac{\pi n^{2}}{t^{2}}} d t,
$$

whence follows

$$
\begin{aligned}
\Re F(x) & =\frac{2 z}{k} \int_{0}^{\infty} \sum_{m=1}^{\infty} \sum_{n=1}^{\infty} e^{-\frac{\pi m^{2} z^{2} t^{2}}{k^{2}}-\frac{\pi n^{2}}{t^{2}}} \cos \frac{2 \pi h m n}{k} d t \\
& =\frac{z}{k} \int_{0}^{\infty} \sum_{m=1}^{\infty} e^{-\frac{\pi m^{2} z^{2} t^{2}}{k^{2}}}\left(\sum_{n=-\infty}^{\infty} e^{-\frac{\pi n^{2}}{t^{2}}} \cos \frac{2 \pi h m n}{k}-1\right) d t \\
& =\frac{z}{k} \int_{0}^{\infty} \sum_{m=1}^{\infty} e^{-\frac{\pi m^{2} z^{2} t^{2}}{k^{2}}}\left(\sum_{r=1}^{k} \cos \frac{2 \pi h m r}{k} \sum_{l=-\infty}^{\infty} e^{-\frac{\pi}{t^{2}}(k l+r)^{2}}-1\right) d t .
\end{aligned}
$$

Now we have, by (2),

$$
\begin{aligned}
& \sum_{l=-\infty}^{\infty} e^{-\frac{\pi}{t^{2}}(k l+r)^{2}}=\sum_{l=-\infty}^{\infty} e^{-\frac{\pi k^{2}}{t^{2}}\left(l+\frac{r}{k}\right)^{2}}=\frac{t}{k} \sum_{n=-\infty}^{\infty} e^{-\frac{\pi n^{2} t^{2}}{k^{2}}} \cos \frac{2 \pi n r}{k} \\
& =\frac{t}{k}\left(2 \sum_{n=1}^{\infty} e^{-\frac{\pi n^{2} t^{2}}{k^{2}}} \cos \frac{2 \pi n r}{k}+1\right) \text {, }
\end{aligned}
$$

and further

$$
\sum_{r=1}^{k} \cos \frac{2 \pi h m r}{k}=\Re \sum_{r=0}^{k-1} e^{\frac{2 \pi i h m r}{k}}=\left\{\begin{array}{l}
k, \text { if } k \mid m, \\
0, \text { otherwise. }
\end{array}\right.
$$

Thus we have

$$
\sum_{m=1}^{\infty} e^{-\pi m^{2} z^{2} t^{2}} \sum_{r=1}^{k} \cos \frac{2 \pi h m r}{k}=k \sum_{m=1}^{\infty} e^{-\pi m^{2} z^{2} t^{2}},
$$

(8) $\quad \Re F(x)=\frac{z}{k} \int_{0}^{\infty}\left(\sum_{m=1}^{\infty} \sum_{n=1}^{\infty} \frac{2 t}{k} e^{-\pi m^{2} z^{2} t^{2}-\pi n^{2} t^{2}} \sum^{k} \sum_{r=1}^{k} \cos \frac{2 \pi h m r}{k} \cos \frac{2 \pi n r}{k}\right.$

$$
\left.+t \sum_{m=1}^{\infty} e^{-\pi m^{2} z^{2} t^{2}}-\sum_{m=1}^{\infty} e^{-\frac{\pi m^{2} z^{2} t^{2}}{k^{2}}}\right) d t
$$


Since we defined

$$
x=e^{\frac{2 \pi i h}{k}-\frac{2 \pi z}{k}}, \quad \tilde{x}=e^{\frac{2 \pi i H}{k}-\frac{2 \pi}{k z}},
$$

it follows from (8) that

$$
\begin{array}{r}
\Re F(\tilde{x})=\frac{1}{k z} \int_{0}^{\infty}\left(\sum_{m=1}^{\infty} \sum_{n=1}^{\infty} \frac{2 t}{k} e^{-\frac{\pi m^{2} t^{2}}{k^{2} z^{2}}-\frac{\pi n^{2} t^{2}}{k^{2}} \sum_{r=1}^{k} \cos \frac{2 \pi H m r}{k} \cos \frac{2 \pi n r}{k}}\right. \\
\left.+t \sum_{m=1}^{\infty} e^{-\frac{\pi m^{2} t^{2}}{z^{2}}}-\sum_{m=1}^{\infty} e^{-\frac{\pi m^{2} t^{2}}{k^{2} z^{2}}}\right) d t \\
=\frac{z}{k} \int_{0}^{\infty}\left(\sum_{m=1}^{\infty} \sum_{n=1}^{\infty} \frac{2 t}{k} e^{-\frac{\pi m^{2} t^{2}}{k^{2}}-\frac{\pi n^{2} z^{2} t^{2}}{k^{2}}} \sum_{r=1}^{k} \cos \frac{2 \pi H m r}{k} \cos \frac{2 \pi n r}{k}\right. \\
\left.+t \sum_{n=1}^{\infty} e^{-\pi n^{2} t^{2}}-\frac{1}{z} \sum_{n=1}^{\infty} e^{-\frac{\pi n^{2} t^{2}}{k^{2}}}\right) d t .
\end{array}
$$

We find here, since $h H \equiv-1(\bmod k)$,

$$
\begin{aligned}
\sum_{r=1}^{k} \cos \frac{2 \pi H m r}{k} \cos \frac{2 \pi n r}{k} & =\sum_{r=1}^{k} \cos \frac{2 \pi H m(h r)}{k} \cos \frac{2 \pi n(h r)}{k} \\
& =\sum_{r=1}^{k} \cos \frac{2 \pi m r}{k} \cos \frac{2 \pi h n r}{k} .
\end{aligned}
$$

Inserting this in the above result and interchanging the indices $m$ and $n$, we obtain

$$
\text { (9) } \begin{gathered}
\Re F(\widetilde{x})=\frac{z}{k} \int_{0}^{\infty}\left(\sum_{m=1}^{\infty} \sum_{n=1}^{\infty} \frac{2 t}{k} e^{-\frac{\pi m^{2} z^{2} t^{2}}{k^{2}}-\frac{\pi n^{2} t^{2}}{k^{2}} \sum_{r=1}^{k} \cos \frac{2 \pi h m r}{k} \cos \frac{2 \pi n r}{k}}\right. \\
+t \sum_{m=1}^{\infty} e^{-\pi m^{2} t^{2}}-\frac{1}{\left.z \sum_{m=1}^{\infty} e^{-\frac{\pi m^{2} t^{2}}{k^{2}}}\right) d t}
\end{gathered}
$$

Subtracting (9) from (8) side-by-side, we get

$$
\begin{aligned}
\Re F(x)-\Re F(\tilde{x}) & \\
= & \frac{z}{k} \int_{0}^{\infty}\left(t \sum_{n=1}^{\infty} e^{-\pi n^{2} z^{2} t^{2}}-\sum_{n=1}^{\infty} e^{-\frac{\pi n^{2} z^{2} t^{2}}{k^{2}}}-t \sum_{n=1}^{\infty} e^{-\pi n^{2} t^{2}}+\frac{1}{z} \sum_{n=1}^{\infty} e^{-\frac{\pi n^{2} t^{2}}{k^{2}}}\right) d t \\
= & \frac{z}{k} \int_{0}^{\infty} t \sum_{n=1}^{\infty} e^{-\pi n^{2} z^{2} t^{2}} d t-\frac{z}{k} \int_{0}^{\infty} t \sum_{n=1}^{\infty} e^{-\pi n^{2} t^{2}} d t \\
& +\frac{1}{k} \int_{0}^{\infty}\left(\sum_{n=1}^{\infty} e^{-\frac{\pi n^{2} t^{2}}{k^{2}}}-z \sum_{n=1}^{\infty} e^{-\frac{\pi n^{2} z^{2} t^{2}}{k^{2}}}\right) d t
\end{aligned}
$$


We have here

$$
\begin{aligned}
& \frac{z}{k} \int_{0}^{\infty} t \sum_{n=1}^{\infty} e^{-\pi n^{2} z^{2} t^{2}} d t=\frac{1}{2 k z} \int_{0}^{\infty} \sum_{n=1}^{\infty} e^{-\pi n^{2} u} d u \\
&=\frac{1}{2 k z} \sum_{n=1}^{\infty} \int_{0}^{\infty} e^{-\pi n^{2} u} d u=\frac{1}{2 \pi k z} \sum_{n=1}^{\infty} \frac{1}{n^{2}}=\frac{\pi}{12 k z}, \\
& \frac{z}{k} \int_{0}^{\infty} t \sum_{n=1}^{\infty} e^{-\pi n^{2} t^{2}} d t=\frac{z}{2 k} \int_{0}^{\infty} \sum_{n=1}^{\infty} e^{-\pi n^{2} u} d u=\frac{z}{2 k \pi} \sum_{n=1}^{\infty} \frac{1}{n^{2}}=\frac{\pi z}{12 k}, \\
& \frac{1}{k} \int_{0}^{\infty}\left(\sum_{n=1}^{\infty} e^{-\frac{\pi n^{2} t^{2}}{k^{2}}}-z \sum_{n=1}^{\infty} e^{-\pi n^{2} z^{2} t^{2}}\right) d t=\int_{0}^{\infty}\left(\sum_{n=1}^{\infty} e^{\left.-\pi n^{2} u^{2}-z \sum_{n=1}^{\infty} e^{-\pi n^{2} z^{2} u^{2}}\right) d u}\right. \\
&=\lim _{\alpha \rightarrow+0}\left(\int_{\alpha}^{\infty} \sum_{n=1}^{\infty} e^{-\pi n^{2} u^{2}} d u-z \int_{\alpha}^{\infty} \sum_{n=1}^{\infty} e^{-\pi n^{2} z^{2} u^{2}} d u\right) \\
&=\lim _{\alpha \rightarrow+0} \int_{\alpha}^{\alpha z} \sum_{n=1}^{\infty} e^{-\pi n^{2} u^{2}} d u .
\end{aligned}
$$

Since we have

$$
\sum_{n=1}^{\infty} e^{-\pi n^{2} t}=\frac{1}{\sqrt{t}} \sum_{n=1}^{\infty} e^{-\frac{\pi n^{2}}{t}}-\frac{1}{2}+\frac{1}{2 \sqrt{t}}
$$

as the special case $\alpha=0$ of (2), we get

$$
\begin{aligned}
\lim _{\alpha \rightarrow+0} \int_{\infty}^{\alpha z} \sum_{n=1}^{\infty} e^{-\pi n^{2} u^{2}} d u=\lim _{\alpha \rightarrow+0} \int_{\alpha}^{\alpha z}\left(\frac{1}{u} \sum_{n=1}^{\infty} e^{-\frac{\pi n^{2}}{u^{2}}}-\frac{1}{2}+\frac{1}{2 u}\right) d u \\
=\lim _{\alpha \rightarrow+0}\left(\frac{1}{2} \log z-\frac{\alpha z-\alpha}{2}+\int_{\infty}^{\alpha z} \frac{1}{u} \sum_{n=1}^{\infty} e^{-\frac{\pi n^{2}}{u^{2}}} d u\right)=\frac{1}{2} \log z .
\end{aligned}
$$

Thus we find finally

$$
\Re F(x)-\Re F(\breve{x})=\frac{\pi}{12 k z}-\frac{\pi z}{12 k}+\frac{1}{2} \log z,
$$

which is equivalent to (5).

We now turn to the proof of (6). We shall first show that

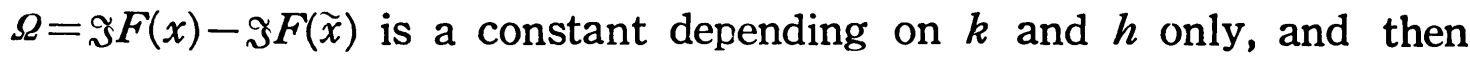
determine the value of $\Omega$ explicitly, using this constancy of $\boldsymbol{\Omega}$.

We have

$$
\frac{d}{d z} \Omega=\Im \frac{d}{d z} F(x)-\Im \frac{d}{d z} F(\widetilde{x})=\Im\left(F^{\prime}(x) \frac{d x}{d z}\right)-\Im\left(F^{\prime}(\widetilde{x}) \frac{d \widetilde{x}}{d z}\right)
$$




$$
=-\mathfrak{s}\left(\frac{2 \pi}{k} x F^{\prime}(x)\right)-\mathfrak{s}\left(\frac{2 \pi}{k z^{2}} \tilde{x} F^{\prime}(\tilde{x})\right) .
$$

Hence, to prove the constancy of $\Omega$, it suffices to show that

$$
z^{2} \Im G(x)+\Im G(\widetilde{x})=0,
$$

where we define, for $|w|<1$,

$$
G(w)=w F^{\prime}(w)=w \sum_{m=1}^{\infty} \sum_{n=1}^{\infty} n w^{m n-1}=\sum_{m=1}^{\infty} \sum_{n=1}^{\infty} n w^{m n} .
$$

We start from

$$
n e^{-\frac{2 \pi m n z}{k}}=\frac{2 z}{k} \int_{0}^{\infty} m n e^{-\frac{\pi m^{2} z^{2} t^{2}}{k^{2}}-\frac{\pi n^{2}}{t^{2}}} d t,
$$

which is equivalent to (7). We find, by absolute convergence, $G(x)=\sum_{m=1}^{\infty} \sum_{n=1}^{\infty} n e^{m n\left(\frac{2 \pi i h}{k}-\frac{2 \pi z}{k}\right)}=\frac{2 z}{k} \int_{0}^{\infty} \sum_{m=1}^{\infty} \sum_{n=1}^{\infty} m n e^{\frac{2 \pi i h m n}{k}-\frac{\pi m^{2} z^{2} t^{2}}{k^{2}}-\frac{\pi n^{2}}{t^{2}}} d t$, and hence

$$
\begin{gathered}
\Im G(x)=\frac{2 z}{k} \int_{0}^{\infty} \sum_{m=1}^{\infty} \sum_{n=1}^{\infty} m n e^{-\frac{\pi m^{2} z^{2} t^{2}}{k^{2}}-\pi^{2} n^{2}} \sin \frac{2 \pi h m n}{k} d t \\
=\frac{z}{k} \int_{0}^{\infty} \sum_{m=1}^{\infty}\left(m e^{-\frac{\pi m^{2} z^{2} t^{2}}{k^{2}}} \sum_{n=-\infty}^{\infty} n e^{-\frac{\pi n^{2}}{t^{2}}} \sin \frac{2 \pi h m n}{k}\right) d t \\
=\frac{z}{k} \int_{0}^{\infty} \sum_{m=1}^{\infty} m e^{-\frac{\pi m^{2} z^{2} \iota^{2}}{k^{2}}} \sum_{r=1}^{k} \sin \frac{2 \pi h m r}{k} \sum_{l=-\infty}^{\infty}(k l+r) e^{-\frac{\pi}{t^{2}}(k l+r)^{2}} d t .
\end{gathered}
$$

Now, differentiating (2) with respect to $\alpha$, we obtain

$$
\sum_{n=-\infty}^{\infty}(n+\alpha) e^{-(n+\alpha)^{2} \pi t}=\frac{2}{t \sqrt{t}} \sum_{n=1}^{\infty} n e^{-\frac{\pi n^{2}}{t}} \sin (2 \pi n \alpha)
$$

and hence

$$
\begin{aligned}
\sum_{l=-\infty}^{\infty}(k l+r) e^{-\frac{\pi}{t^{2}}(k l+r)^{2}} & =k \sum_{l=-\infty}^{\infty}\left(l+\frac{r}{k}\right) e^{-\frac{\pi k^{2}}{t^{2}}\left(l+\frac{r}{k}\right)^{2}} \\
& =\frac{2 t^{3}}{k^{2}} \sum_{n=1}^{\infty} n e^{-\frac{\pi n^{2} t^{2}}{k^{2}}} \sin \frac{2 \pi n r}{k},
\end{aligned}
$$

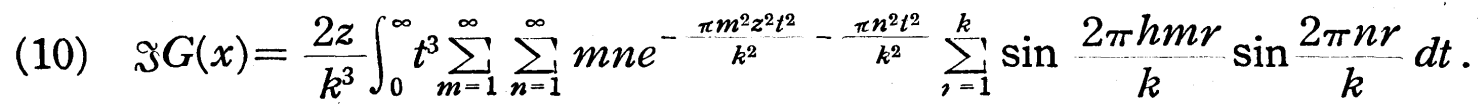


Changing here $z$ to $1 / z$ and $h$ to $H$, we derive

$$
\begin{aligned}
& \Im G(\widetilde{x})=\frac{2}{k^{3} z} \int_{0}^{\infty} t^{3} \sum_{m=1}^{\infty} \sum_{n=1}^{\infty} m n e^{-\frac{\pi m^{2} t^{2}}{k^{2} z^{2}}-\frac{\pi n^{2} t^{2}}{k^{2}}} \sum_{r=1}^{k} \sin \frac{2 \pi H m r}{k} \sin \frac{2 \pi n r}{k} d t
\end{aligned}
$$

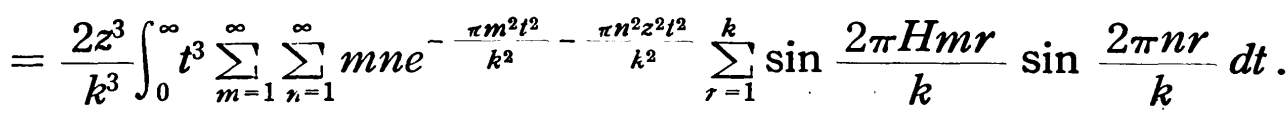

We find here, since $h H \equiv-1(\bmod k)$,

$$
\begin{aligned}
\sum_{r=1}^{k} \sin \frac{2 \pi H m r}{k} \sin \frac{2 \pi n r}{k} & =\sum_{r=1}^{k} \sin \frac{2 \pi H m(h r)}{k} \sin \frac{2 \pi n(h r)}{k} \\
& =-\sum_{r=1}^{k} \sin \frac{2 \pi m r}{k} \sin \frac{2 \pi h n r}{k} .
\end{aligned}
$$

Inserting this in the above result and interchanging the indices $m$ and $n$, we get

$$
\Im G(\widetilde{x})=-\frac{2 z^{3}}{k^{3}} \int_{0}^{\infty} t^{3} \sum_{m=1}^{\infty} \sum_{n=1}^{\infty} m n e^{-\frac{\pi m^{2} 2^{2} t^{2}}{k^{2}}-\frac{\pi n^{2} t^{2}}{k^{2}}} \sum_{r=1}^{k} \sin \frac{2 \pi h m r}{k} \sin \frac{2 \pi n r}{k} d t .
$$

A comparison of the last equality with (10) yields

$$
\Im G(\widetilde{x})=-z^{2} \Im G(x),
$$

which was to be proved.

We shall now deduce the formula (4) for $\Omega$. The following proof of (4) is substantially that given in Dedekind [1], Dedekind omits, however, the verification of the uniform convergence of a certain series which, though easy to establish, forms the kernel of his proof; we shall give, in the following, a detailed discussion on this uniformity.

We may assume $k>1$. For if $k=1$ both $x$ and $\tilde{x}$ are real and we have

$$
\Omega=\Im F(x)-\Im F(\widetilde{x})=0-0=0,
$$

while the expression (4) also vanishes for $k=1$.

We have, by (6), assuming $z>0$ as hitherto,

$$
\lim _{z \rightarrow 0} \Im F(x)=\Omega+\lim _{z \rightarrow 0} \Im F(\widetilde{x})=\Omega+\Im F(0)=\Omega .
$$

Let us put

$$
R=e^{-\frac{2 \pi z}{k}}(z>0), \quad \rho=e^{\frac{2 \pi i h}{k}},
$$


so that $x=R \rho$. Then

$$
\begin{gathered}
F(x)=\sum_{m=1}^{\infty} \sum_{n=1}^{\infty} \frac{x^{m n}}{m}=\sum_{n=1}^{\infty} \frac{1}{n} \frac{x^{n}}{1-x^{n}}, \\
\Im F(x)=\sum_{n=1}^{\infty} \frac{1}{n} \Im\left(\frac{x^{n}}{1-x^{n}}\right)=\sum_{n=1}^{\infty} \frac{1}{n} \Im\left(\frac{1}{1-x^{n}}\right)=\sum_{n=0}^{\infty} A_{n}(R),
\end{gathered}
$$

where we define, for $n=0,1,2, \cdots$,

$$
A_{n}(R)=\sum_{r=1}^{k-1} \frac{1}{r+k n} \Im\left(\frac{1}{1-\rho^{r} R^{r+k n}}\right)
$$

We put further, for $n=1,2,3, \cdots$,

$$
B_{n}(R)=\sum_{r=1}^{k-1} \frac{1}{k n} \Im\left(\frac{1}{1-\rho^{r} R^{r^{+k n}}}\right)
$$

Then

$$
\begin{aligned}
\left|A_{n}(R)-B_{n}(R)\right| & \leqq \sum_{r=1}^{k-1} \frac{r}{k n(r+k n)}\left|\Im\left(\frac{1}{1-\rho^{r} R^{r+k n}}\right)\right| \\
& \leqq \sum_{r=1}^{k-1} \frac{r}{k n(r+k n)} \frac{1}{\left|1-\rho^{r} R^{r+k n}\right|^{2}} .
\end{aligned}
$$

We now define, for real positive $y$ and natural numbers $r<k$,

$$
\lambda(y)=\left|1-\rho^{r} y\right|^{2}=1-2 y \cos 2 \theta+y^{2}=(y-\cos 2 \theta)^{2}+\sin ^{2} 2 \theta,
$$

where we use the abbreviation $\theta=\pi h r / k$. We find then, if $\cos 2 \theta \leqq 0$,

$$
\lambda(y) \geq 1+y^{2}>1,
$$

and if $\cos 2 \theta>0$, then we have $k \geqq 4$ (since $k=2$ or $k=3$ would imply $\cos 2 \theta<0$ against the hypothesis), and hence

$$
\lambda(y) \geq \sin ^{2} 2 \theta \geq \sin ^{2} \frac{2 \pi}{k} \geq \sin ^{2} \frac{\pi}{k}>\frac{1}{k^{2}} .
$$

We thus derive in both cases

$$
\left|1-\rho^{r} y\right|^{2}>\frac{1}{k^{2}}
$$

and this combined with the above inequality gives

$$
\left|A_{n}(R)-B_{n}(R)\right| \leqq \frac{k^{2}}{n^{2}} \quad(n=1,2,3, \cdots) .
$$


Let us define further

$$
C_{n}(R)=\sum_{r=1}^{k-1} \frac{1}{k n} \Im\left(\frac{1}{1-\rho^{r} R^{k n}}\right) \quad(n=1,2,3, \cdots) .
$$

Then we have

$$
C_{n}(R)=\sum_{,=1}^{k-1} \frac{1}{k n} \Im\left(\frac{1}{1-\rho^{k-r} R^{k n}}\right)=\sum_{r=1}^{k-1} \frac{1}{k n} \Im\left(\frac{1}{1-\rho^{-r} R^{k n}}\right) .
$$

Adding this to (16) side-by-side, we find

$$
2 C_{n}(R)=\sum_{r=1}^{k-1} \frac{1}{k n} \Im\left(\frac{1}{1-\rho^{r} R^{k n}}+\frac{1}{1-\rho^{-r} R^{k n}}\right)=0 ;
$$

therefore, subtracting (16) from (13) and noting (14), we obtain

$$
\begin{aligned}
\left|B_{n}(R)\right| & \leqq \sum_{r=1}^{k-1} \frac{1}{k n}\left|\frac{1}{1-\rho^{r} R^{r+k n}}-\frac{1}{1-\rho^{r} R^{k n}}\right| \\
& \leqq \sum_{r=1}^{k-1} \frac{1}{k n} \frac{R^{k n}\left(1-R^{r}\right)}{\left|1-\rho^{r} R^{r+k n}\right| \cdot\left|1-\rho^{r} R^{k n}\right|} \\
& \leqq \frac{k}{n} R^{k n}(1-R) \sum_{r=1}^{k-1} \sum_{m=0}^{r-1} R^{m} \leqq \frac{k^{3}}{n} R^{k n}(1-R) .
\end{aligned}
$$

Since the unique root of

$$
\frac{d}{d y} y^{k n}(1-y)=k n y^{k n-1}-(k n+1) y^{k n}=0
$$

in the interval $0<y<1$ is given by $y=k n /(k n+1)$, at which point the function $y^{k n}(1-y)$ takes its maximum in $0<y<1$, we have

$$
\left|B_{n}(R)\right| \leqq \frac{k^{3}}{n} \frac{1}{k n+1}<\frac{k^{2}}{n^{2}} .
$$

It follows from (15) and the last inequality that

$$
\left|A_{n}(R)\right|<\frac{2 k^{2}}{n^{2}} \quad(n=1,2,3, \cdots) .
$$

Hence $\sum_{n=0}^{\infty} A_{n}(R)$ is uniformly convergent with respect to $R$ in the interval $0<R<1$. We have therefore, noting (11) and (12),

$$
\Omega=\lim _{R \rightarrow 1} \sum_{n=0}^{\infty} A_{n}(R)=\sum_{n=0}^{\infty} A_{n}(1)
$$




$$
=\sum_{n=0}^{\infty} \sum_{r=1}^{k-1} \frac{1}{r+k n} \mathfrak{\Im}\left(\frac{1}{1-\rho^{r}}\right)=\frac{1}{2} \sum_{n=0}^{\infty} \sum_{r=1}^{k-1} \frac{1}{r+k n} \cot \frac{\pi h r}{k} .
$$

We find, on the other hand, since

$$
\begin{aligned}
& t-[t]-\frac{1}{2}=-\sum_{n=1}^{\infty} \frac{\sin 2 \pi n t}{\pi n} \quad(t \text { real and not integer }), \\
& \sum_{m=1}^{k-1} 2 m \sin \theta \sin 2 m \theta=\sum_{m=1}^{k} 2 m \sin \theta \sin 2 m \theta \quad(\theta=\pi h r / k) \\
= & \sum_{m=1}^{k}(m \cos (2 m-1) \theta-m \cos (2 m+1) \theta)=-k \cos \theta,
\end{aligned}
$$

the following result:

$$
\begin{aligned}
\pi \sum_{m=1}^{k-1} \frac{m}{k}\left(\frac{h m}{k}-\left[\frac{h m}{k}\right]-\frac{1}{2}\right)=-\pi \sum_{m=1}^{k-1} \frac{m}{k} \sum_{n=1}^{\infty} \frac{1}{\pi n} \sin \frac{2 \pi h m n}{k} \\
=-\sum_{n=1}^{\infty} \frac{1}{k n} \sum_{m=1}^{k-1} m \sin \frac{2 \pi h m n}{k}=-\sum_{n=0}^{\infty} \sum_{r=1}^{k-1} \frac{1}{k(r+k n)} \sum_{m=1}^{k-1} m \sin \frac{2 \pi h m r}{k} \\
=\frac{1}{2} \sum_{n=0}^{\infty} \sum_{r=1}^{k-1} \frac{1}{r+k n} \cot \frac{\pi h r}{k},
\end{aligned}
$$

and comparing this with (17), we find the required formula for the case $k>1$.

The rest of the present paper is devoted to the study of two properties of $\Omega$.

First, we continue from (17) and derive for $k>1$

$$
\Omega=-\frac{1}{2} \sum_{n=1}^{\infty} \sum_{r=1}^{k-1} \frac{1}{k n-r} \cot \frac{\pi h r}{k},
$$

which added to (17) side-by-side yields

$$
\begin{aligned}
2 \Omega & =\sum_{r=1}^{k-1} \frac{1}{2 r} \cot \frac{\pi h r}{k}-\sum_{r=1}^{k-1} \cot \frac{\pi h r}{k} \sum_{n=1}^{\infty} \frac{r}{k^{2} n^{2}-r^{2}} \\
& =\frac{1}{2} \sum_{r=1}^{k-1}\left(\cot \frac{\pi h r}{k}\right)\left(\frac{1}{r}-\sum_{n=1}^{\infty} \frac{2 r}{k^{2} n^{2}-r^{2}}\right) .
\end{aligned}
$$

Since

$$
\cot s=\frac{1}{s}-\sum_{n=1}^{\infty} \frac{2 s}{\pi^{2} n^{2}-s^{2}} \quad(|s|<\pi),
$$


we have

$$
\cot \frac{\pi r}{k}=\frac{k}{\pi}\left(\frac{1}{r}-\sum_{n=1}^{\infty} \frac{2 r}{k^{2} n^{2}-r^{2}}\right),
$$

and hence we get

$$
\Omega_{h, k}=\frac{\pi}{4 k} \sum_{m=1}^{k-1} \cot \frac{\pi h m}{k} \cot \frac{\pi m}{k} \quad(k>1),
$$

which also holds for $k=1$, if an empty sum signifies zero. We have thus found two different expressions (4) and (19) for $\Omega$.

Secondly, our arithmetic function $\Omega_{h, k}$ is known to satisfy a curious equality called the reciprocity formula for Dedekind sums, which asserts that, for coprime positive integers $h$ and $k$,

$$
\sum_{m=1}^{k-1} \frac{m}{k}\left\{\frac{h m}{k}\right\}+\sum_{n=1}^{h-1} \frac{n}{h}\left\{\frac{k n}{h}\right\}=\frac{h^{2}+k^{2}+1}{12 h k}-\frac{1}{4},
$$

where we use the abbreviation $\{t\}=t-[t]-\frac{1}{2}$ for real $t$.

Rademacher and Whiteman [5] gave a simple arithmetic proof for (20). We shall give, in the following, a simple contour-integration proof for

(21) $\frac{1}{k} \sum_{m=1}^{k-1} \cot \frac{\pi h m}{k} \cot \frac{\pi m}{k}+\frac{1}{h} \sum_{n=1}^{h-1} \cot \frac{\pi k n}{h} \cot \frac{\pi n}{h}=\frac{h^{2}+k^{2}+1}{3 h k}-1$, which is equivalent to (20) on account of (19) and (4).

We put, for a complex variable $z=x+i y$ ( $x$ and $y$ real),

$$
g(z)=\cot z \cot \frac{z}{k} \cot \frac{h z}{k} .
$$

We take $\alpha>\frac{1}{2}$ and define four paths of integration as follows : The path $A$ begins at $\alpha i$, thence goes vertically down to $(1 / 2) i$, then runs along the semi-circle $z=(1 / 2) e^{i \theta},(1 / 2)_{\pi} \leqq \theta \leqq(3 / 2)_{\pi}$ with origin the centre counter-clockwise, and finally descends from $-(1 / 2) i$ down to $-\alpha i$; the path $B$ starts at $-\alpha i$, proceeds horizontally to the right and ends at $k_{\pi}-\alpha i$; we name $C$ the path which begins at $k_{\pi}-\alpha i$, ascends vertically up to $k \pi-(1 / 2) i$, then runs clockwise along the semi-circle $z=k \pi+(1 / 2) e^{i \theta},(3 / 2)_{\pi} \geqq \theta \geqq(1 / 2)_{\pi}$, and finally proceeds from $k \pi+(1 / 2) i$ vertically upwards, terminating at $k \pi+\alpha i$; the last 
path $D$ originates at $k \pi+\alpha i$, goes horizontally to the left and ends at $\alpha i$.

We name $K$ the contour obtained by successive junctions of the paths just obtained. Then, integrating once round $K$ in the positive sense, we get

$$
\int_{K} g(z) d z=\int_{A}+\int_{B}+\int_{C}+\int_{D},
$$

which equals $2 \pi i$ times the sum of the residues of $g(z)$ at the poles inside $K$.

Since $g(z)$ has a period $k \pi$, we have

$$
\int_{A}+\int_{C}=0 \text {. }
$$

We find also, uniformly in $x$,

$$
\cot z \rightarrow \mp i \quad(y \rightarrow \pm \infty),
$$

and hence, as $\alpha$ tends to infinity,

$$
\int_{B}+\int_{D} \rightarrow \int_{0}^{k \pi}(-i) d x-\int_{0}^{k \pi} i d x=-2 k \pi i
$$

Now we have, by (18) for instance, the expansion

$$
z \cot z=1-\frac{1}{3} z^{2}+\cdots \quad(|z|<\pi),
$$

and hence, for small $|z|$,

$$
(z \cot z)\left(\frac{z}{k} \cot \frac{z}{k}\right)\left(\frac{h z}{k} \cot \frac{h z}{k}\right)=1-\frac{h^{2}+k^{2}+1}{3 k^{2}} z^{2}+\cdots .
$$

Hence the residue of $g(z)$ at the origin is $-\left(h^{2}+k^{2}+1\right) / 3 h$. Further, all the poles of $g(z)$ inside $K$ and different from the origin are $\pi m(m=1,2, \cdots, k-1)$ in the case $k>1$, and $(k / h)_{\pi} n(n=1,2, \cdots, h-1)$ in the case $h>1$, with the residues

$$
\cot \frac{\pi h m}{k} \cot \frac{\pi m}{k} \quad \text { and } \quad \frac{k}{h} \cot \frac{\pi k n}{h} \cot \frac{\pi n}{h}
$$

respectively. Hence we find, making $\alpha \rightarrow \infty$,

$$
\sum_{m=1}^{k-1} \cot \frac{\pi h m}{k} \cot \frac{\pi m}{k}+\frac{k}{h} \sum_{n=1}^{h-1} \cot \frac{\pi k n}{h} \cot \frac{\pi n}{h}=\frac{h^{2}+k^{2}+1}{3 h}-k,
$$

which is equivalent to (21). 


\section{References}

[1] R. Dedekind: Erläuterungen zu den Fragmenten XXVIII ; Bernhard Riemanns Gesammelte Mathematische Werke und Wissenschaftlicher Nachlass (2. Auflage, Leipzig 1892), pp. 466-478.

[2] G. H. Hardy and S. Ramanujan: Asymptotic formulae in combinatory analysis ; Proc. London Math. Soc. (2), vol. 17 (1918), pp. 75-115.

[3] H. Rademacher: Zur Theorie der Modulfunktionen; J. Reine Angew. Math. vol. 167 (1932), pp. 312-336.

[4] H. Rademacher: On the partition function $p(n)$; Proc. Iondon Math. Soc. (2), vo'. 43 (1937), pp. 241-254.

[5] H. Rademacher and A. Whiteman: Theorems on Dedekind sums; Amer. J. Math. vol. 63 (1941), pp. 377-407.

\section{Related papers}

1) T. M. Apostol: Generalized Dedekind sums and transformation formulae of certain Lambert series; Duke Math. J. vol. 17 (1950), pp. 147-157.

2) T.M. Apostol: Asymptotic series related to the partition function; Ann. of Math. (2), vol. 53 (1951), pp. 327-331.

3) R. Bellman: Ramanujan sums and the average value of arithmetic functions; Duke Math. J. vol. 17 (1950), pp. 159-168.

4) N. A. Brigham: A general asymptotic formula for partition functions; Proc. Amer. Math. Soc. vol. 1 (1950), pp. 182-191.

5) N. A. Brigham: On a certain weighted partition function; Proc. Amer. Math. Soc. vol. 1 (1950), pp. 192-204.

6) C. Hermite: Sur quelques formules relatives à la transformation des functions elliptiques; J. de Math. (2), vol. 3 (1858), pp. 26-36.

7) H. Rademacher: Bestimmung einer gewissen Einheitswurzel in der Theorie der Modulfunktionen; J. London Math. Soc. vol. 7 (1932), pp. 14-19.

8) H. Rademacher: On the expansion of the partition function in a series: Ann. of Math. (2), vol. 44 (1943), pp. 416-422.

9) H. Rademacher: Die Reziprozitätsformel für Dedekindsche Summen; Acta Sci. Math. Szeged 12, Leopoldo Fejér et Frederisco Riesz LXX annos natis dedicatus, pars B, pp. 57-60 (1950).

10) L. Rédei : Elementarer Beweis und Verallgemeinerung einer Reziprozitätsformel von Dedekind; Acta Sci. Math. Szeged 12, Leopoldo Fejér et Frederisco Riesz LXX annos natis dedicatus, pars B, pp. 236-239 (1950).

11) L. Schoenfeld: A transformation formula in the theory of partitions; Duke Math. J. vol. 11 (1944), pp. 873-887. 